\title{
Avaliação do desempenho de sistemas alagados construídos de escoamento horizontal subsuperficial tratando efluente de reator UASB, com base em quatro anos de monitoramento
}

\author{
Performance evaluation of horizontal subsurface flow constructed wetlands \\ in the treatment of UASB reactor effluent, based on four years of monitoring \\ Jocilene Ferreira Costa', André Oliveira De Paoli², Marcos Von Sperling ${ }^{3}$, Martin Seidl ${ }^{4}$
}

\begin{abstract}
RESUMO
Um sistema composto por um reator anaeróbio tipo upflow anaerobic sludge blanket (UASB), seguido de um sistema de alagados construídos (SAC) de escoamento horizontal subsuperficial compreendendo duas unidades em paralelo (50 habitantes cada), foiavaliado para otratamento deáguas residuárias geradas na cidade de Belo Horizonte, Minas Gerais, Brasil. Uma unidade foi plantada com Typha latifolia e a outra não foi vegetada. Amostras semanais do efluente do reator UASB e do efluente das unidades do SAC foram avaliadas por um período de quatro anos de operação do sistema. Nas taxas de aplicação praticadas (média de $0,11 \mathrm{~m}^{3} \cdot \mathrm{m}^{2} \cdot \mathrm{d}^{-1}$ ), o efluente final apresentou excelente qualidade em termos de matéria orgânica e sólidos suspensos, mas baixa capacidade de remoção de nitrogênio. Os valores médios de concentração no efluente das unidades plantadas e não plantadas foram, respectivamente, demanda bioquímica de oxigênio: 25 e 23 mg.L L'ㄹ demanda química de oxigênio:

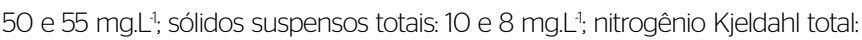

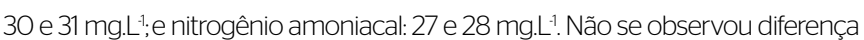
significativa entre as concentrações efluentes das unidades plantada e não plantada para a maioria dos constituintes investigados.
\end{abstract}

Palavras-chave: Wetlands construídas; sistemas alagados construídos; reator UASB; águas residuárias domésticas; Typha latifolia.

\begin{abstract}
A system composed of an upflow anaerobic sludge blanket (UASB) reactor followed by two horizontal subsurface flow wetlands (50 inhabitants each) constructed in parallel was evaluated for the treatment of municipal wastewater generated in the city of Belo Horizonte, Minas Gerais, Brazil. One unit was planted with Typha latifolia, and the other one was not planted. Weekly samples of the effluent from the UASB reactor and the effluent from both wetland units were evaluated for a period of four years of the system's operation. With the applied loads (average of $0.11 \mathrm{~m}^{3} \cdot \mathrm{m}^{-2} \cdot \mathrm{d}^{-1}$ ), the final effluent showed excellent quality in terms of organic matter and suspended solids, but it showed low capacity for nitrogen removal. Mean concentration values in the effluent from the planted and unplanted units were, respectively, biochemical oxygen demand: 25 and 23 mg. $\mathrm{L}^{-1}$; chemical oxygen demand: 50 and 55 mg. $\mathrm{L}^{-1}$; total suspended solids: 10 and 8 mg. L $^{-1}$; total Kjeldahl nitrogen: 30 and 31 mg. L $^{-1}$; and N-ammonia: 27 and $28 \mathrm{mg}^{-1} \mathrm{~L}^{-1}$. There was no significant difference between the effluent concentrations from the planted and unplanted units for most of the evaluated constituents.
\end{abstract}

Keywords: constructed wetlands; constructed subsurface flow; UASB reactor; domestic wastewater; Typha latifolia.

\section{INTRODUÇÃO}

Atualmente, existem muitas tecnologias de tratamento para a recuperação e manutenção da integridade física, química e biológica das águas. Durante os últimos 20 anos, considerável interesse tem sido dedicado ao potencial de uso de uma variedade de sistemas biológicos naturais para ajudar na purificação da água de maneira controlada. A utilização de plantas no tratamento de esgoto representa uma tecnologia emergente, eficiente, estética e de baixos custos energéticos, que está

'Doutora em Saneamento, Meio Ambiente e Recursos Hídricos pela Universidade Federal de Minas Gerais (UFMG) - Belo Horizonte (MG), Brasil. ${ }^{2}$ Mestre em Saneamento, Meio Ambiente e Recursos Hídricos pela UFMG - Belo Horizonte (MG), Brasil.

${ }^{3}$ Doutor pelo Imperial College. Professor Titular do Departamento de Engenharia Sanitária e Ambiental da UFMG - Belo Horizonte (MG), Brasil.

${ }^{4}$ Doutor em Ciências Ambientais pela Escola Nacional de Engenharia Civil da Universidade de Paris. Pesquisador do Laboratoire Eau Environnement et Systèmes Urbanis (França) e UFMG - Belo Horizonte (MG), Brasil.

Endereço para correspondência: Jocilene Ferreira Costa - Avenida Antonio Carlos, n. 6.627 - Escola de Engenharia, bloco 1, sala 46220 - Pampulha - $31270-901$ - Belo Horizonte (MG), Brasil - E-mail: jocilenefc@bol.com.br

Recebido em: 06/05/2012 - Aceito em: 24/01/2017 - Reg. ABES: 90370 
se revelando uma boa alternativa aos sistemas convencionais mecanizados (VICZNEVSKI; MARCHESINI, 2002; PRESZNHUK et al., 2003; ALMEIDA et al., 2005).

Sistemas alagados construídos (SACs) são uma tecnologia de tratamento de águas residuárias baseada nos processos encontrados em ecossistemas das várzeas naturais, podendo ser classificados como um sistema natural. Uma das principais variantes é representada pelos sistemas alagados construídos de escoamento horizontal subsuperficial (SAC-EHSS), nos quais podem predominar condições anaeróbias ou anóxicas pelo fato de os espaços vazios do leito serem preenchidos por esgotos em tratamento. Na zona anóxica dos SAC-EHSS pode ocorrer a redução do nitrato a nitrogênio gasoso pelas bactérias heterotróficas, além da conversão da matéria orgânica (SCHULZ, 2009). Para Davies et al. (2004), na zona anaeróbia, a eficiência de remoção de demanda bioquímica de oxigênio (DBO) ocorre devido à elevada capacidade de decomposição de bactérias anaeróbias presentes.

Todos os mecanismos nos SACs ocorrem de modo concomitante e influenciados pelo tipo de escoamento, pela planta cultivada, pelo meio suporte utilizado e pelas características da água residuária a ser tratada, sendo que a grande maioria dos poluentes fica aderida ao sedimento e às plantas (USEPA, 1999).

No tratamento de esgotos domésticos, SACs têm sido eficientes como uma etapa de polimento de vários sistemas de tratamento de esgotos, principalmente para a remoção complementar da matéria orgânica e de sólidos. Em estudos na Austrália, a média de eficiência de sistemas de wetlands tratando efluentes domésticos atingiu $81 \mathrm{e}$ $83 \%$ para demanda bioquímica de oxigênio (DBO) e sólidos suspensos totais (SST), respectivamente (DAVISON; HEADLEY; PRATT, 2004). No Canadá, Boutilier et al. (2009), investigando SACs cultivados com Typha latifolia, foi obtida eficiência de remoção de cerca $76 \%$ de SST, utilizando tempos de detenção hidráulica de 25 dias. Já na República Tcheca, Vymazal (2004) evidenciou, em sua pesquisa, eficiência de remoção de 81,89 e 91\% para demanda química de oxigênio (DQO), DBO e SST, respectivamente, utilizando Typha sp. e também tratando esgoto doméstico. Em regiões de clima semiárido, como na Espanha, Solano, Soriano e Ciria (2004) encontraram remoções de 63 a 93\%, 50 a 88\% e 58 a 94\% para DQO, DBO e SST, respectivamente, utilizando taxas de aplicação hidráulica de 0,150 e $0,075 \mathrm{~m}^{3} \cdot \mathrm{m}^{-2} \cdot \mathrm{d}^{-1} \mathrm{e}$ dois tipos de macrófitas, Typha sp. e Phragmites sp. Baskar, Deeptha e Annadurai (2014), em seu estudo em SACs na região sul da Índia, com clima tropical e precipitação média anual de $1.330 \mathrm{~mm}$ e utilizando efluentes domésticos pré-tratados, tendo como plantas Typha latifolia e Phragmites australis, substrato cascalho e areia, encontraram percentuais de redução de DBO de 23 a 45\%, com tempo de detenção hidráulica (TDH) variando entre 2 e 8 dias. No Brasil, em Campinas, São Paulo, foram obtidas, por Valentim (2003), remoções de até 77 e $81 \%$ para DQO e SST, respectivamente, tendo como meio suporte do SACs brita \#2, utilizando 3 espécies de macrófitas emergentes - Typha sp., Eleocharis sp. e Scirpus sp. - e TDH de 1 a 6 dias.

No Brasil, a tecnologia atual de tratamento de esgotos tem usualmente envolvido uma primeira etapa com reatores anaeróbios, seguida por uma de polimento, podendo ser usados vários processos de tratamento com tal finalidade. Com relação à utilização de SAC-EHSS como pós-tratamento de efluentes anaeróbios, alguns trabalhos podem ser encontrados na literatura, mas há necessidade de mais informações consolidadas de longo prazo em unidades funcionando em escala real. Sousa et al. (2004) avaliaram, durante três anos de monitoramento, o desempenho de SAC-EHSS recebendo efluente de reator tipo upflow anaerobic sludge blanket (UASB), no Nordeste do Brasil. Nesse sistema, a remoção de DQO variou de 70 a $86 \%$ de eficiência. Para o nitrogênio Kjeldahl total (NTK) e fósforo $(\mathrm{P})$ total, este tipo de tratamento foi mais eficiente no primeiro ano de operação, atingindo 66 a 86\% de remoção, respectivamente. Bastos et al. (2010), também investigando unidades de SAC como pós-tratamento, submetidas a distintas taxas de aplicação, obtiveram, em todas as condições operacionais, DBO e sólidos suspensos (SS) inferiores a $25 \mathrm{mg} . \mathrm{L}^{-1}$. Resultados obtidos por Mbuligwe (2004), na Tanzânia, demonstraram que SACs horizontais subsuperficiais cultivados com Typha podem efetivamente remover fósforo, sulfato, amônia e DQO de esgoto doméstico pré-tratado em reatores UASB. As remoções médias alcançadas nas unidades foram de 69 a $75 \%$ para fósforo, 72 a 77\% para sulfato, 74 a $75 \%$ para amônia e 75 a $79 \%$ para DQO. Kaseva (2004), estudando o desempenho de um SAC-EHSS como pós-tratamento de reator UASB, também na Tanzânia, obteve eficiência de remoção de DQO de 61\% (Typha sp).

Segundo Mander et al. (2004), a remoção de nitrogênio em SAC ocorre principalmente por meio dos processos de nitrificação e desnitrificação, sendo os microrganismos os principais responsáveis por essas transformações. A eficiência de remoção de nitrogênio tem sido pouco expressiva e muito variada (VALENTIM, 2003). Mander et al. (2004) obtiveram valores de taxas de remoção de nitrogênio variando de 7,4 a 18,9 g.m $\mathrm{m}^{-2} \cdot$ ano $^{-1}$ na biomassa aérea.

O presente estudo tem como objetivo avaliar o desempenho na remoção de matéria orgânica, sólidos e nitrogênio de esgoto sanitário oriundo de um reator UASB em um sistema alagado construído de escoamento horizontal subsuperficial, com uma unidade plantada (Typha latifolia) e outra não plantada com macrófitas, com base em cerca de quatro anos de operação. A relevância está associada à necessidade de se ampliar as pesquisas com esse tipo de pós-tratamento de efluentes de reatores anaeróbios. Ademais, trata-se de uma avaliação de longo termo, o que complementa informações sobre o comportamento de sistemas de alagados construídos, uma vez que a maioria dos trabalhos com esse tipo de processo é feita em curtos prazos, o que não 
permite uma avaliação consolidada do seu desempenho (KADLEC; WALLACE, 2009).

\section{MATERIAIS E MÉTODOS}

A unidade experimental do sistema alagado construído de escoamento horizontal subsuperficial utilizada para o pós-tratamento de esgoto doméstico por reator UASB encontra-se no Centro de Pesquisa e Treinamento em Saneamento (CePTS) da Universidade Federal de Minas Gerais (UFMG), Companhia de Saneamento de Minas Gerais (COPASA), localizado na Estação de Tratamento de Esgoto (ETE) do Arrudas, na cidade de Belo Horizonte, Brasil. A montante do sistema experimental, o esgoto recebe tratamento preliminar, composto por gradeamento mecanizado (grade grossa e fina) e desarenador da própria ETE-Arrudas.

O sistema de tratamento objeto deste estudo é constituído por um reator UASB que foi projetado para tratar uma vazão de $30 \mathrm{~m}^{3} \cdot \mathrm{d}^{-1} \mathrm{de}$ efluente coletado no canal de esgoto bruto, seguido por duas unidades em paralelo de SAC com leito de escória siderúrgica de escoamento horizontal subsuperficial.

Cada unidade do SAC foi dimensionada para um equivalente populacional de cerca de 50 habitantes e para uma vazão contínua e constante de $7,5 \mathrm{~m}^{3} \cdot \mathrm{d}^{-1}$. A granulometria do leito de escória é caracterizada por $\mathrm{d}_{10}=19 \mathrm{~mm}$ e o coeficiente de desuniformidade $\mathrm{d}_{60} / \mathrm{d}_{10}=1,2$. A altura do leito filtrante é $0,40 \mathrm{~m}$, sendo que a lâmina d'água de projeto possui 0,30 m (DORNELAS, 2008). Um leito foi cultivado com taboa (Typha latifolia), respeitando densidade inicial de quatro plantas por metro quadrado, e o outro leito não foi plantado, atuando como controle. O corte da vegetação foi realizado manualmente, sempre que necessário, por meio de cortes rasos, após o florescimento das plantas e antes do início da dispersão das sementes. As principais dimensões e características de projeto e dados operacionais do SAC encontram-se na Tabela 1, e uma vista geral das unidades é apresentada na Figura 1.

$\mathrm{Na}$ entrada das unidades do SAC foram dispostas "pedras de mão" com granulometria entre 10 e $15 \mathrm{~cm}$ de diâmetro, com a zona de entrada tendo dimensões de 3,0 m largura $\times 0,4 \mathrm{~m}$ altura $\times 0,5 \mathrm{~m}$ comprimento, formando um leito, com a finalidade da distribuição uniforme do efluente e da contenção de sólidos provenientes do pré-tratamento.

O monitoramento do sistema foi realizado de setembro de 2007 a agosto de 2011 por meio de amostragens simples do esgoto bruto, do efluente do reator UASB e das saídas das unidades do SAC. Para o presente trabalho foram avaliados os seguintes parâmetros físico-químicos nas amostras de efluentes: $\mathrm{DQO}, \mathrm{DBO}_{5}$, sólidos totais, NTK e nitrogênio amoniacal, além da vazão. A frequência de monitoramento foi semanal e também foram efetuadas amostragens para análise de
DQO filtrada ao longo do perfil longitudinal das unidades, com as coletas sendo realizadas em 5 pontos distintos ao longo do comprimento das unidades (0, 25, 50, 75 e 100\% da dimensão horizontal). As análises dos parâmetros de qualidade foram realizadas no Laboratório de Análises Físico-químicas do Departamento de Engenharia Sanitária e Ambiental da UFMG, de acordo com os procedimentos constantes no Standard Methods for the Examination of Water and Wastewater (APHA; AWWA; WEF, 2005).

Os dados de precipitação pluviométrica utilizados no estudo foram obtidos por meio da estação meteorológica localizada no Parque Mangabeiras, em Belo Horizonte, Minas Gerais.

A análise estatística dos dados para comparar o desempenho entre as unidades plantada (UP) e não plantada (UNP) foi realizada pelo

Tabela 1 - Principais características de projeto e de operação para cada unidade do sistema de alagados construídos.

\begin{tabular}{l|c|c|c} 
Parâmetro & Unidade & $\begin{array}{c}\text { Valor de } \\
\text { projeto }\end{array}$ & $\begin{array}{c}\text { Valor médio dos } \\
\text { quatro anos de } \\
\text { operação }\end{array}$ \\
\hline Altura total dos leitos & $\mathrm{m}$ & 0,4 & 0,4 \\
\hline Altura do líquido nos leitos & $\mathrm{m}$ & 0,3 & 0,3 \\
\hline Comprimento & $\mathrm{m}$ & 24,1 & 24,1 \\
\hline Largura & $\mathrm{m}$ & 3,0 & 3,0 \\
\hline Área superficial & $\mathrm{m}^{2}$ & 72,3 & 72,3 \\
\hline Volume útil (molhado) & $\mathrm{m}^{3}$ & 21,7 & 21,7 \\
\hline $\begin{array}{l}\text { TAH média } \\
\text { (Q/área superficial) }\end{array}$ & $\mathrm{m}^{3} \cdot \mathrm{m}^{-2} \cdot \mathrm{d}^{-1}$ & 0,12 & 0,11 \\
\hline $\begin{array}{l}\text { TDH média } \\
\text { (volume } \times \text { porosidade/Q) }\end{array}$ & $\mathrm{d}$ & 1,2 & 1,1 \\
\hline Vazão média & $\mathrm{m}^{3} \cdot \mathrm{d}^{-1}$ & 7,5 & 8,0 \\
\hline
\end{tabular}

TAH: taxa de aplicação hidráulica; Q: vazão; TDH: tempo de detenção hidráulica.

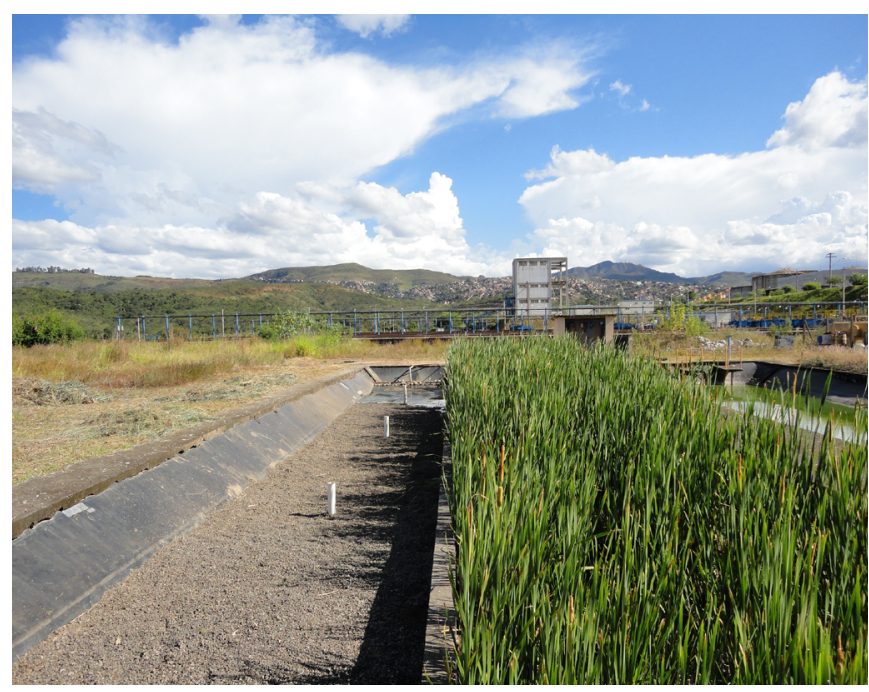

Figura 1 - Vista das unidades do sistema de alagados construídos, não plantada e plantada. 
teste não paramétrico entre variáveis dependentes Wilcoxon a 5\% de significância, empregando o software Statistica .

\section{RESULTADOS E DISCUSSÃO}

Na Tabela 2 são apresentados os resultados das vazões médias afluentes e efluentes obtidas durante o período de quatro anos de operação do sistema.

Pode-se observar, pela Tabela 2, que as vazões efluentes dos dois sistemas são menores que as afluentes, além da unidade vegetada ter apresentado vazão efluente média menor que a não plantada. Tal fato se deve à evaporação em ambas e à transpiração que ocorre apenas no cultivado. Acredita-se que as perdas por infiltração não sejam importantes nos dois sistemas, já que o fundo de cada um foi impermeabilizado com argila compactada com $0,20 \mathrm{~m}$ de espessura, os taludes foram revestidos com geomembrana de polietileno de alta densidade (PEAD) e o muro divisório de alvenaria foi coberto por camada impermeabilizada. A Figura 2 apresenta as médias mensais de perdas de água por evapotranspiração e transpiração nas UP e UNP, juntamente com as médias mensais de precipitação. Observa-se que a cultivada sempre teve maior perda de água que a não plantada, e que as maiores perdas ocorreram nos meses secos, com menos precipitações médias.

Tabela 2 - Médias das vazões afluente e efluente de cada unidade do sistema de alagados construídos.

\begin{tabular}{l|c|c|c} 
Unidade & $\begin{array}{c}\text { Vazão afluente } \\
\text { média }\left(\mathrm{m}^{3} \cdot \mathrm{d}^{-1}\right)\end{array}$ & $\begin{array}{c}\text { Vazão efluente } \\
\text { média }\left(\mathrm{m}^{3} \cdot \mathrm{d}^{-1}\right)\end{array}$ & $\begin{array}{c}\text { Perda média de } \\
\text { água (\%) }\end{array}$ \\
\hline Plantada & 8,2 & 6,6 & 19,5 \\
\hline Não plantada & 8,0 & 6,8 & 15,0 \\
\hline
\end{tabular}

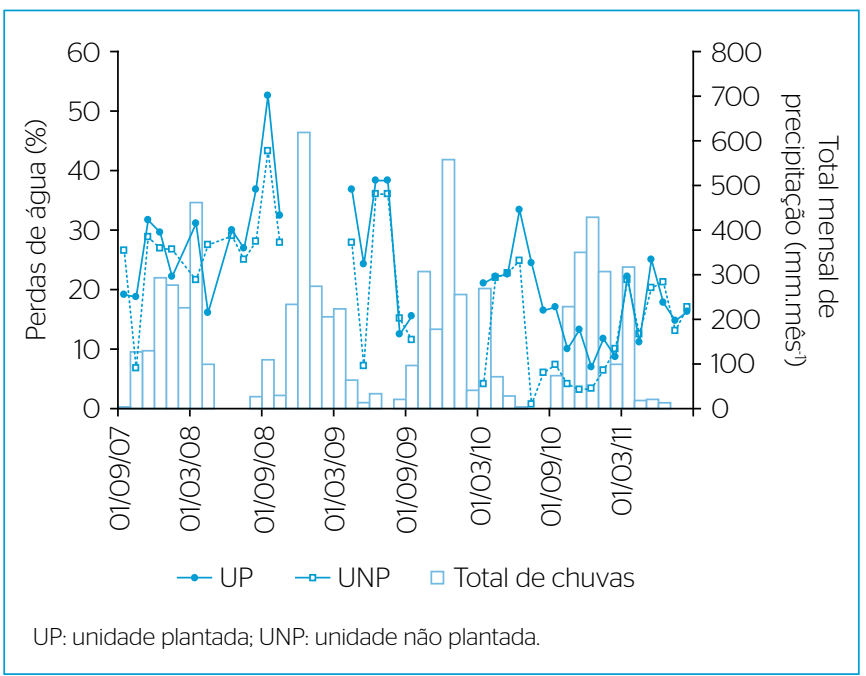

Figura 2 - Médias mensais de perda de água por evapotranspiração e evaporação no sistema de alagados construídos, juntamente com os totais mensais de precipitação.
Blanc, Quirion e Shobl (2008) relatam que o aumento da evapotranspiração decorre da elevação da demanda evaporativa da atmosfera, dentre outros fatores, e é causado pela crescente radiação solar global. Esse fenômeno segue um ciclo diário que depende diretamente da temperatura do ar, da radiação e exposição solar. As perdas por evapotranspiração reduzem o volume de água, aumentando a concentração dos poluentes, o que não conduz, no entanto, a um aumento da carga destes no efluente do sistema (RIBEIRO, 2007). Se for o caso, a concentração efluente pode ser corrigida em função das perdas de água, por meio da Equação 1.

Concentração $=$ concentração medida $\times(1-$ fração de perda de água $)(1)$

Na Tabela 3, encontram-se os valores das concentrações médias efluentes tais como foram medidas e com os valores considerando as perdas de água em cada unidade do SAC (aplicando a Equação 1). Observa-se que a inclusão da perda de água leva a menores concentrações no efluente, o que retrata mais proximamente o real comportamento dos SACs. No entanto, no restante do trabalho são apresentadas as concentrações sem considerar a perda de água, pelo fato de serem as mais usuais, e por permitirem melhor comparação com os valores reportados na literatura.

A Tabela 4 apresenta os dados relativos à estatística descritiva dos parâmetros correspondentes à matéria orgânica $\left(\mathrm{DBO}_{5} \mathrm{e} \mathrm{DQO}\right)$ e sólidos (SST) para o efluente do reator UASB e efluentes do SAC das UP e UNP (sem correção para a perda de água). A Tabela 5 tem estrutura similar, apresentando as estatísticas descritivas das principais frações de nitrogênio. Observa-se a excelente qualidade do efluente final, em termos de matéria orgânica e sólidos em suspensão, em ambas as unidades do SAC, mas há concentrações ainda relativamente elevadas de NTK e N amoniacal. Deve-se destacar também que o reator UASB gera um efluente de boa qualidade em termos de matéria orgânica e sólidos, o que propicia a obtenção de baixas concentrações no esgoto final.

Tabela 3 - Concentração média efluente medida e valores corrigidos em função das perdas de água em cada unidade do sistema de alagados construídos.

\begin{tabular}{c|c|c|c|c}
\multirow{2}{*}{ Parâmetros* } & \multicolumn{2}{|c|}{ Unidade plantada } & \multicolumn{2}{c}{ Unidade não plantada } \\
\cline { 2 - 5 } & $\begin{array}{c}\text { Concentração } \\
\text { medida }\end{array}$ & $\begin{array}{c}\text { Concentração } \\
\text { considerando } \\
\text { perda de água }\end{array}$ & $\begin{array}{c}\text { Concentração } \\
\text { medida }\end{array}$ & $\begin{array}{c}\text { Concentração } \\
\text { considerando } \\
\text { a perda de } \\
\text { água }\end{array}$ \\
\hline DQO & 50 & 40 & 55 & 47 \\
\hline DBO & 25 & 20 & 23 & 18 \\
\hline SST & 8 & 6 & 9 & 8 \\
\hline NTK & 30 & 24 & 31 & 26 \\
\hline N amoniacal & 27 & 22 & 28 & 24 \\
\hline
\end{tabular}

DQO: demanda química de oxigênio; DBO: demanda bioquímica de oxigênio; SST: sólidos suspensos totais; NTK: nitrogênio Kjeldahl total; *perdas de água médias: unidade plantada (19,5\%), unidade não plantada (15,0\%). 
As médias das eficiências de remoção nas UP e UNP, calculadas em termos de concentração (sem correção pelas perdas de água, ou seja, segundo a forma de cálculo tradicional) e em termos de carga (concentração afluente versus vazão afluente e concentração efluente versus vazão efluente, ou seja, levando em consideração as perdas de água), encontram-se na Tabela 6. Observa-se que a determinação da eficiência com base na carga conduz a valores superiores aos da eficiência com base na concentração, representando, assim, a real participação do sistema na remoção dos poluentes. De fato, ao se analisar os valores de eficiência dos SACs com base na carga removida, observam-se excelentes resultados, compatíveis com sistemas mais avançados de tratamento.

Tabela 4 - Estatística descritiva das concentrações efluentes de demanda química de oxigênio, demanda bioquímica de oxigênio e sólidos suspensos totais.

\begin{tabular}{|c|c|c|c|c|c|c|c|c|c|}
\hline \multirow{2}{*}{ Estatística* } & \multicolumn{3}{|c|}{ UASB } & \multicolumn{3}{|c|}{ Unidade plantada } & \multicolumn{3}{|c|}{ Unidade não plantada } \\
\hline & DQO & DBO & SST & DQO & DBO & SST & DQO & DBO & SST \\
\hline Número de dados & 107 & 99 & 66 & 111 & 94 & 76 & 109 & 97 & 75 \\
\hline Média & 179 & 83 & 60 & 50 & 25 & 8 & 55 & 23 & 9 \\
\hline Mediana & 164 & 71 & 43 & 50 & 20 & 8 & 52 & 19 & 6 \\
\hline Mínimo & 50 & 21 & 14 & 11 & 5 & 1,1 & 4 & 5 & 0,6 \\
\hline Máximo & 456 & 368 & 262 & 106 & 90 & 23,5 & 139 & 103 & 75 \\
\hline Desvio-padrão & 75 & 58 & 50,5 & 19 & 19 & 5 & 25 & 17 & 11 \\
\hline Coeficiente de variação & 0,42 & 0,70 & 1,33 & 0,40 & 0,80 & 0,73 & 0,45 & 0,75 & 1,02 \\
\hline
\end{tabular}

*Exceto para número de dados e coeficiente de variação (ambos adimensionais), a unidade é mg.L'1; UASB: upflow anaerobic s/udge blanket; DQO: demanda química de oxigênio; DBO: demanda bioquímica de oxigênio; SST: sólidos suspensos totais.

Tabela 5 - Estatística descritiva das concentrações efluentes de nitrogênio Kjeldahl total e amoniacal.

\begin{tabular}{l|c|c|c|c|c|c}
\multirow{2}{*}{ Estatística* } & \multicolumn{2}{|c|}{ UASB } & \multicolumn{2}{c}{ Unidade plantada } & Unidade não plantada \\
\cline { 2 - 7 } Número de dados & NTK & N amoniacal & NTK & N amoniacal & NTK & 126 \\
\hline Média & 132 & 131 & 122 & 122 & 31 \\
\hline Mediana & 32 & 29 & 30 & 27 & 31 & 28 \\
\hline Mínimo & 33 & 29 & 29 & 27 & 7 \\
\hline Máximo & 6 & 12 & 55 & 54 & 52 & 52 \\
\hline Desvio-padrão & 55 & 52 & 40 & 37 & 0 \\
\hline Coeficiente de variação & 40 & 31 & 0,31 & 0,36 & 0,28 & 0 \\
\hline
\end{tabular}

*Exceto para número de dados e coeficiente de variação (ambos adimensionais), a unidade é mg.L'1'; UASB: upflow anaerobic s/udge blanket; NTK: nitrogênio Kjeldahl total.

Tabela 6 - Eficiências médias de remoção nas unidades plantada e não plantada do sistema de alagados construídos.

\begin{tabular}{|c|c|c|c|c|c|c|c|}
\hline \multirow[t]{2}{*}{ Parâmetros } & \multirow{2}{*}{$\begin{array}{c}\text { Eficiência (\%) } \\
\text { UASB }\end{array}$} & \multicolumn{2}{|c|}{$\begin{array}{l}\text { Eficiência do sistema de alagados } \\
\text { construídos com base na } \\
\text { concentração removida (\%) }\end{array}$} & \multicolumn{2}{|c|}{$\begin{array}{l}\text { Eficiência do sistema de } \\
\text { alagados construídos com } \\
\text { base na carga removida (\%) }\end{array}$} & \multicolumn{2}{|c|}{$\begin{array}{l}\text { Eficiência global do sistema, com } \\
\text { base na concentração removida (\%) }\end{array}$} \\
\hline & & Up & UNP & UP & UNP & $(\mathrm{UASB}+\mathrm{UP})$ & (UASB+UNP) \\
\hline DQO & 57 & 72 & 69 & 93 & 92 & 88 & 87 \\
\hline DBO & 72 & 70 & 72 & 92 & 93 & 91 & 92 \\
\hline SST & 81 & 87 & 85 & 97 & 97 & 97 & 97 \\
\hline NTK & - & 6 & 3 & 30 & 15 & - & - \\
\hline N amoniacal & - & 7 & 3 & 30 & 15 & - & - \\
\hline
\end{tabular}

UASB: upflow anaerobic sludge blanket; UP: unidade plantada; UNP: unidade não plantada; DQO: demanda química de oxigênio; DBO: demanda bioquímica de oxigênio; SST: sólidos suspensos totais; NTK: nitrogênio Kjeldahl total. Eficiências calculadas com base nas concentrações e vazões médias afluente e efluente; eficiência expressa em concentração: (concentração afluente - concentração efluente)/concentração afluente; eficiência expressa em carga: (carga afluente - carga efluente)/carga afluente. 
Pode-se observar uma boa eficiência, em cada etapa do tratamento, para a remoção de matéria orgânica e sólidos. O reator UASB proporcionou uma remoção média de 57, 72 e 81\% de DQO, DBO e SST, respectivamente. As duas unidades de SAC, mesmo atuando como pós-tratamento, contribuíram com uma elevada remoção complementar, conduzindo a altas eficiências globais de remoção.

Souza et al. (2002) avaliaram por três anos dois SACs tratando efluente de reator UASB. Uma unidade era plantada com Juncus $s p$ e outra servia como controle. Os autores encontraram eficiências de remoção de DQO variando de 70 a $86 \%$, e de SST entre 50 e $71 \%$. Já Valentim (2003) obteve resultados de eficiência de remoção de 70\% de DQO no tratamento de águas residuárias domésticas em SACs cultivados com taboa. E Toniato et al. (2005), também estudando SAC como pós-tratamento de sistema anaeróbios, encontraram valores de DBO entre 4 e 79 mg.L $\mathrm{L}^{-1}$ e eficiência de remoção de $67 \%$.

Para N amoniacal, a remoção média em termos de carga foi de $30 \mathrm{e}$ 15\% para a UP e UNP, respectivamente, ao longo dos 4 anos de operação do sistema estudado. Esses valores são superiores ao encontrado por Rios et al. (2009), que, avaliando SAC-EHSS, obtiveram eficiência média em função da carga de $22 \%$ de remoção de amônia. Por outro lado, Mbuligwe (2004) obteve resultados maiores, de 74 e 63\% para UP e UNP, respectivamente, operando como pós-tratamento de UASB. Essa análise de remoção dos parâmetros, quando baseada na diferença da carga afluente e efluente, ressalta ainda mais o melhor desempenho geral para a UP.

$\mathrm{Na}$ Figura 3 encontram-se as séries temporais das concentrações efluentes de DQO, DBO e SST do reator UASB e da UP e UNP do SAC.

No primeiro ano de funcionamento do SAC houve menor concentração de SST no efluente, percebendo-se ligeiras elevações na concentração ao final do período estudado, possivelmente evidenciando o aumento nas concentrações afluentes e/ou o processo de colmatação do sistema. O aumento das concentrações afluentes aos SAC ocorreu devido a problemas de corrosão no separador trifásico do reator UASB, o que ocasionou grandes perdas de sólidos. Nessa mesma fase encontram-se as maiores concentrações de matéria orgânica (DBO), o que demonstra a interligação desses dois parâmetros (DBO e SST), podendo ter ocorrido, no período, um carreamento orgânico maior.

$\mathrm{Na}$ Figura 4 são apresentadas, na forma de gráfico box plot, as concentrações efluentes da UP e UNP. Pelos gráficos, pode-se avaliar o atendimento aos padrões de lançamento do estado de Minas Gerais — Deliberação Normativa do Conselho Estadual de Política Ambiental - Conselho de Recursos Hídricos nº 01 - (MINAS GERAIS, 2008), para DQO:

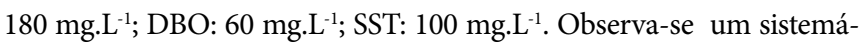
tico atendimento aos padrões de DQO e SST e um elevado atendimento ao padrão de DBO. Verifica-se, ainda, que os valores das concentrações finais poderiam cumprir metas de qualidade ou padrões de lançamento de efluentes mais restritivos. Portanto, o excelente desempenho do SAC de escoamento horizontal subsuperficial na remoção de matéria orgânica e

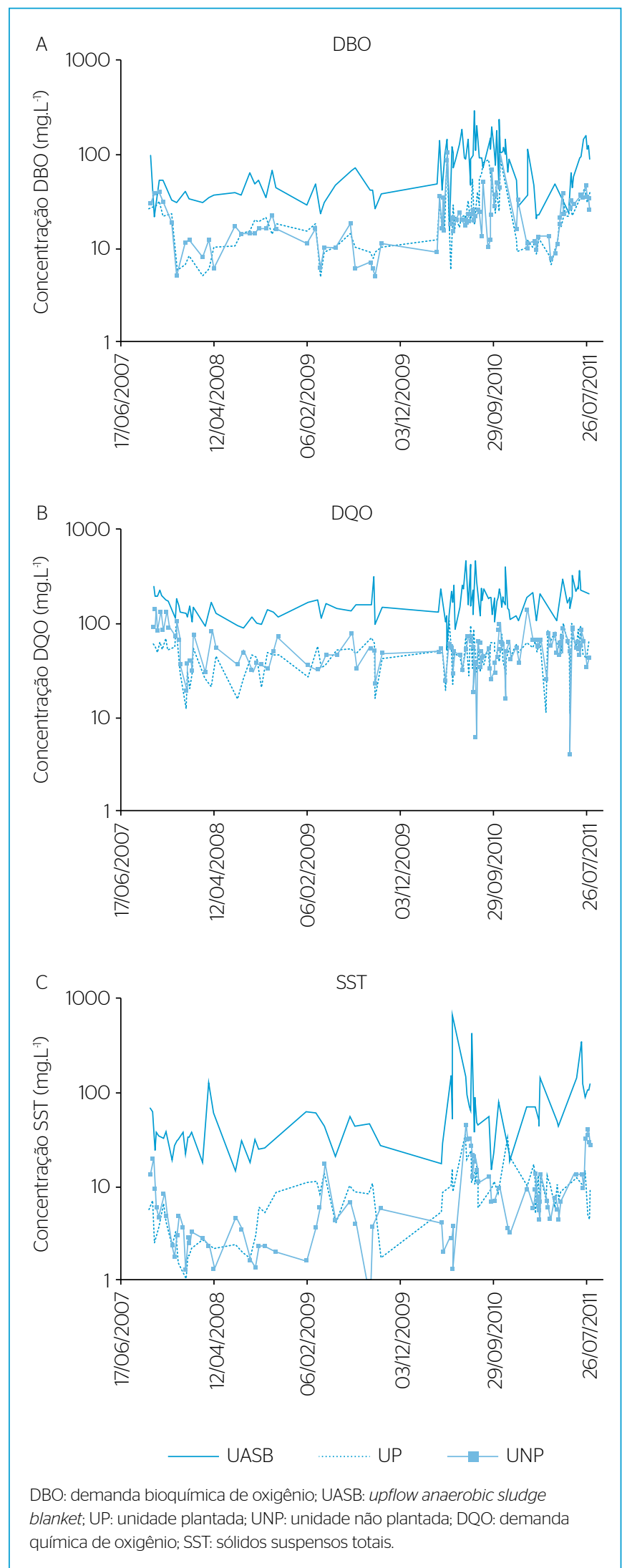

Figura 3 - Séries temporais das concentrações efluentes de (A) demanda química de oxigênio, (B) demanda bioquímica de oxigênio e (C) sólidos suspenso totais para o reator UASB e as unidades do sistema de alagados construídos, planta e não planta. 
sólidos suspensos reportado na literatura é endossado pelos resultados obtidos no sistema em investigação para DBO, DQO e SST nas duas unidades.

A capacidade de remoção de DBO e DQO deve-se ao desenvolvimento da biomassa ao longo do leito. De Paoli e von Sperling (2011), avaliando previamente os sólidos no sistema em uso no presente trabalho,

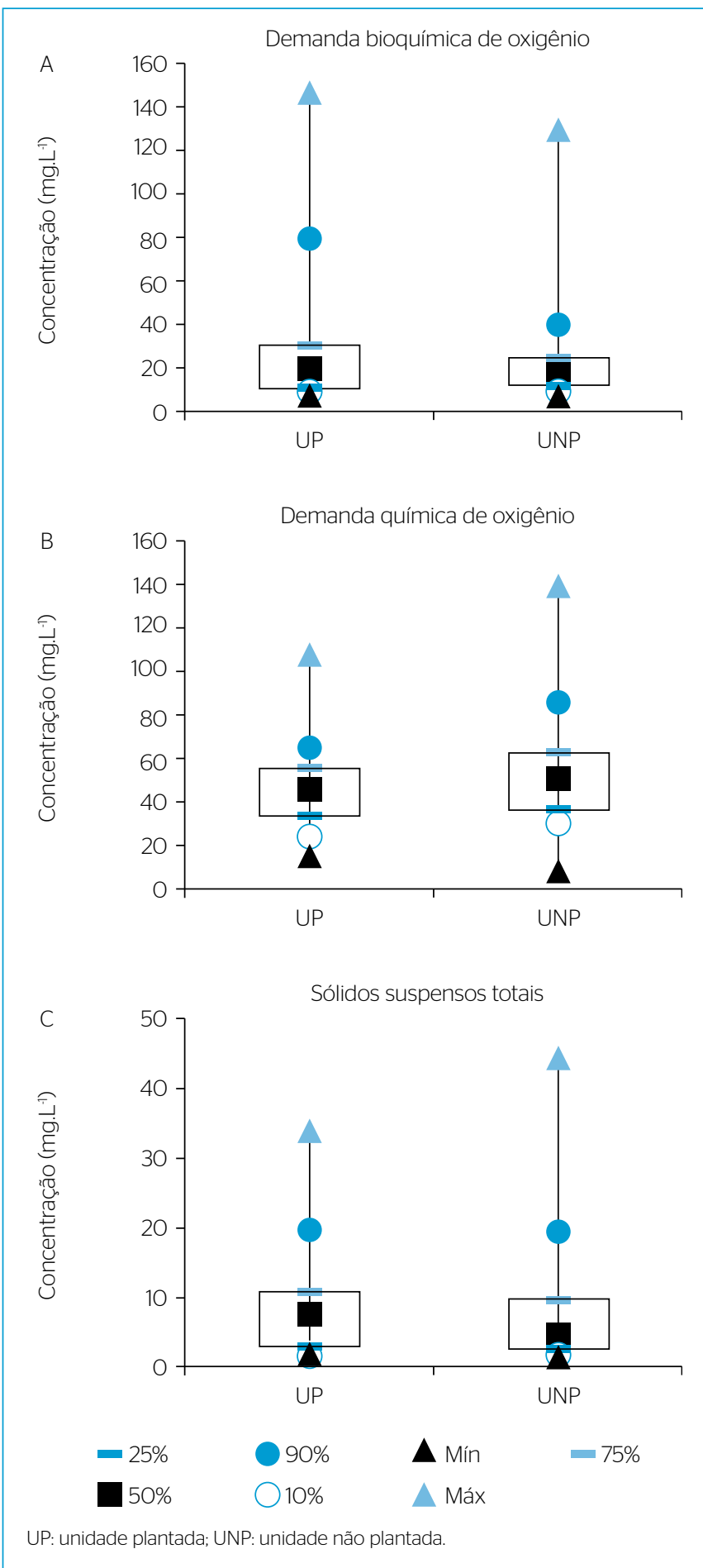

Figura 4 - Box plot das concentrações efluentes de (A) demanda bioquímica de oxigênio, (B) demanda química de oxigênio e (C) sólidos suspensos totais nas unidades plantada e não plantada. concluíram que a maior parte dos sólidos acumulados no leito filtrante é inorgânica (baixa relação sólidos voláteis totais e sólidos totais - SVT/ ST), e também que a maior parte da biomassa se apresenta na forma de sólidos intersticiais, e não na de sólidos aderidos (biofilme).

Avaliou-se ainda o decaimento da matéria orgânica ao longo do percurso longitudinal das unidades. A Figura 5 mostra o gráfico box plot $(n=22)$, com medidas de concentrações de DQO (filtrada) em 5 pontos de amostragem ao longo do percurso longitudinal das 2 unidades do SAC. É visível um decaimento sequencial da matéria orgânica ao longo do comprimento das unidades no formato típico da cinética de primeira ordem. O ajuste do modelo de fluxo em pistão aos valores médios de DQO em cada ponto de amostragem conduziu aos do coeficiente de remoção de DQO de $0,81 \mathrm{~d}^{-1}$ e $0,84 \mathrm{~d}^{-1}$ e do coeficiente de determinação de 0,971 e 0,940 para a UP e UNP, respectivamente. Esses valores estão dentro da faixa usual relatada na literatura citada por De Paoli e von Sperling (2010): 0,8 a 1,1 d ${ }^{-1}$ (REED; CRITES;

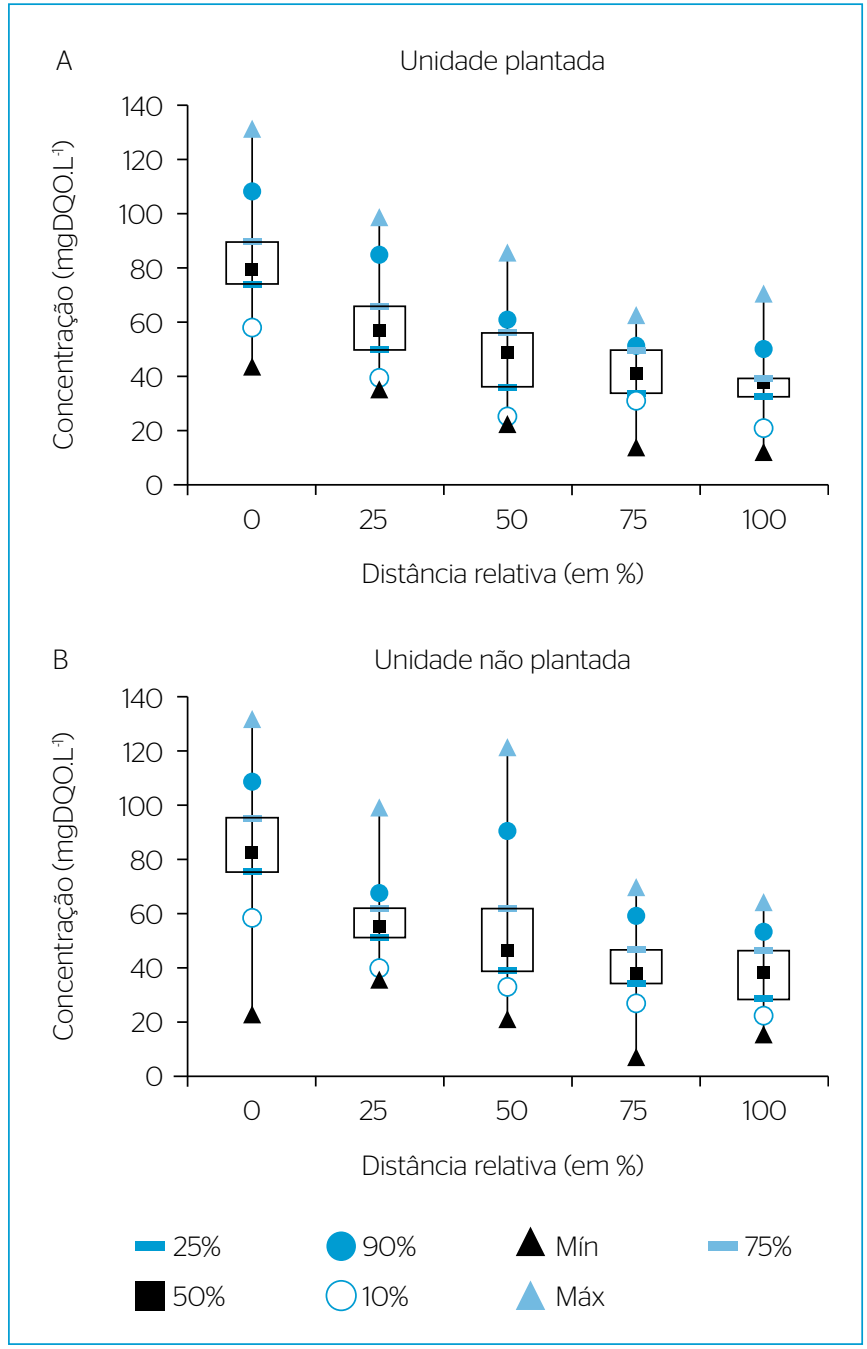

Figura 5 - Box plot de medidas de concentrações de demanda química de oxigênio filtrada ao longo do comprimento das unidades do sistema de alagados construídos (A) plantada e (B) não plantada. 
MIDDLEBROOKS, 1988); 0,7 d $\mathrm{d}^{-1} \pm 0.2$ (CONLEY; DICK; LIOW, 1991); assim como METCALF; EDDY (1991) mencionaram, 0,86 a 1,84 d $\mathrm{d}^{-1}$; 0,17 a 6,11 d $\mathrm{d}^{-1}$ (ROUSSEAU; VABRIKKEGHEM; PAUW, 2004); 0,44 $\mathrm{d}^{-1}$ (BRASIL, 2005); 0,1 a 2,65 d $\mathrm{d}^{-1}$ (STEIN et al., 2006); 0,46 a 0,75 $\mathrm{d}^{-1}$ (SANDOVAL-COBO; PEÑA, 2007); 0,56 a 1,37 d-1 (FIA, 2009).

A Figura 6 mostra o gráfico box plot dos parâmetros referentes às espécies de nitrogênio (Kjeldahl e amoniacal) para o efluente do reator UASB e efluentes na UP e UNP. Conforme já comentado, a remoção dessas espécies nitrogenadas foi muito baixa. O pequeno decréscimo nas concentrações de amônia sugere nitrificação pouco expressiva.

Por meio do teste não paramétrico entre variáveis dependentes Wilcoxon a 5\% de significância (Tabela 7), foi constatado que não houve diferença significativa nas concentrações efluentes entre A UP e UNP para a maior parte dos constituintes (DQO, DBO, SST e NTK).

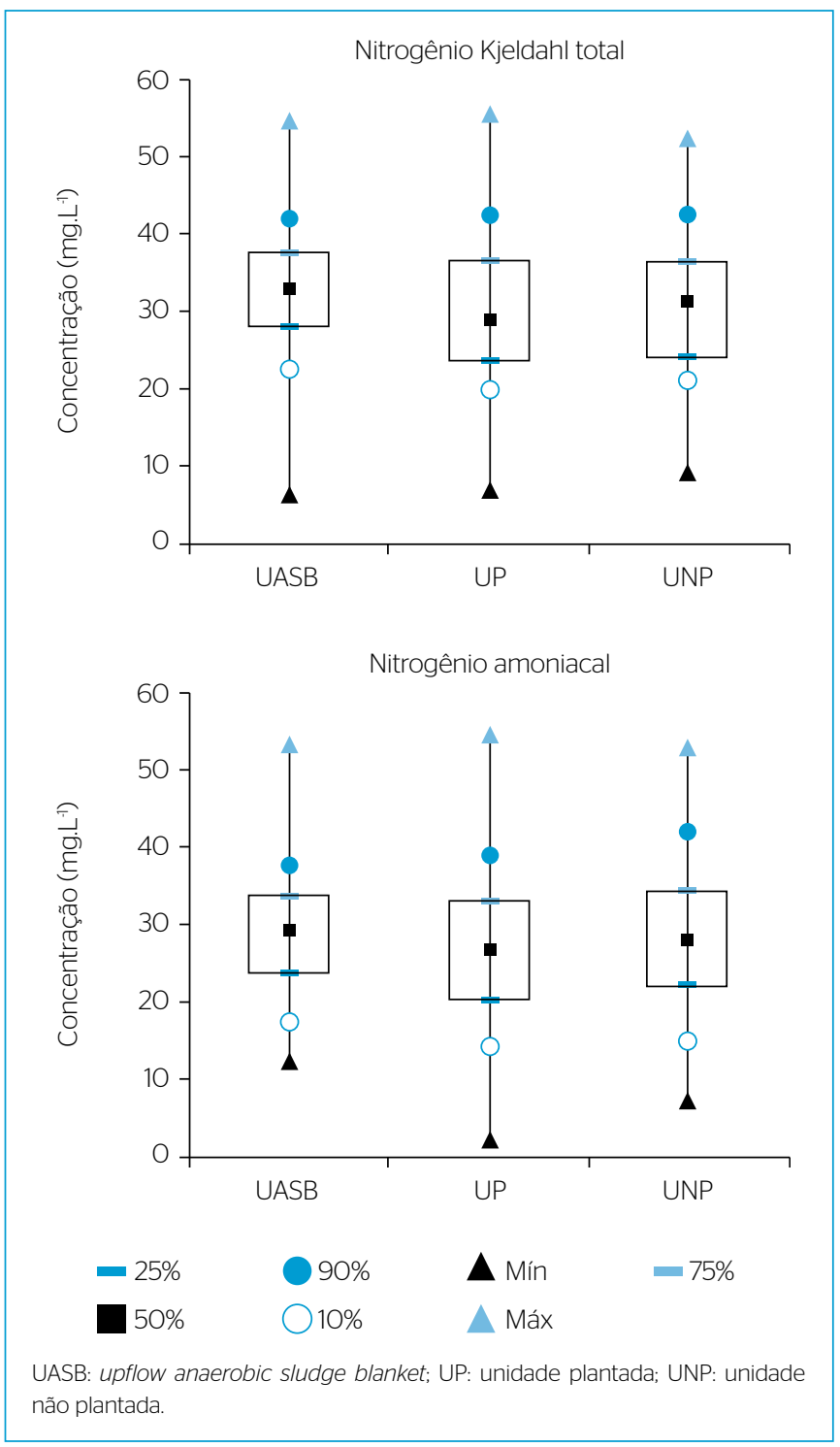

Figura 6 - Box plot das concentrações efluentes de (A) nitrogênio Kjeldahl total e (B) nitrogênio amoniacal do reator UASB e das unidades plantada e não plantada.
Um dos grandes problemas operacionais dos SACs de escoamento subsuperficial relaciona-se à colmatação, que gera aumento da perda de carga e redução da condutividade hidráulica, e pode eventualmente conduzir ao escoamento superficial a partir do início do leito. É evidente o maior avanço do escoamento superficial na UP (Figura 7), fato que pode ter ocorrido devido às raízes da Typha latifolia contribuírem para a criação de biomassa em seu entorno, fazendo com que haja maior colmatação. De fato, na primeira metade do leito, a concentração de sólidos totais da UP foi aproximadamente o dobro da ocorrida na UNP (DE PAOLI, 2010). A colmatação foi bastante acelerada em um período em que o reator UASB estava em obras de manutenção, com substituição do separador trifásico. Nesse período, houve uma elevada perda de sólidos, os quais ficaram retidos nas unidades do SAC.

Tabela 7 - Resultado do teste de Wilcoxon para as medianas das concentrações efluentes das unidades plantada e não plantada.

\begin{tabular}{l|c|c}
\hline Parâmetros & Valor $\mathbf{p}^{*}$ & $\begin{array}{c}\text { Significativamente } \\
\text { diferente? }\end{array}$ \\
\hline DBO & 0,9850 & Não \\
\hline DQO & 0,0559 & Não \\
\hline SST & 0,9527 & Não \\
\hline NTK & 0,0507 & Não \\
\hline N amoniacal & 0,0005 & Sim
\end{tabular}

DBO: demanda bioquímica de oxigênio; DQO: demanda química de oxigênio; SST: sólidos suspensos totais; NTK: nitrogênio Kjeldahl total.

${ }^{*} p<0,05$ : medianas das concentrações efluentes das unidades plantada e não plantada são significativamente diferentes; $p \geq 0,05$ : medianas das concentrações efluentes das unidades plantada e não plantada não são significativamente diferentes.

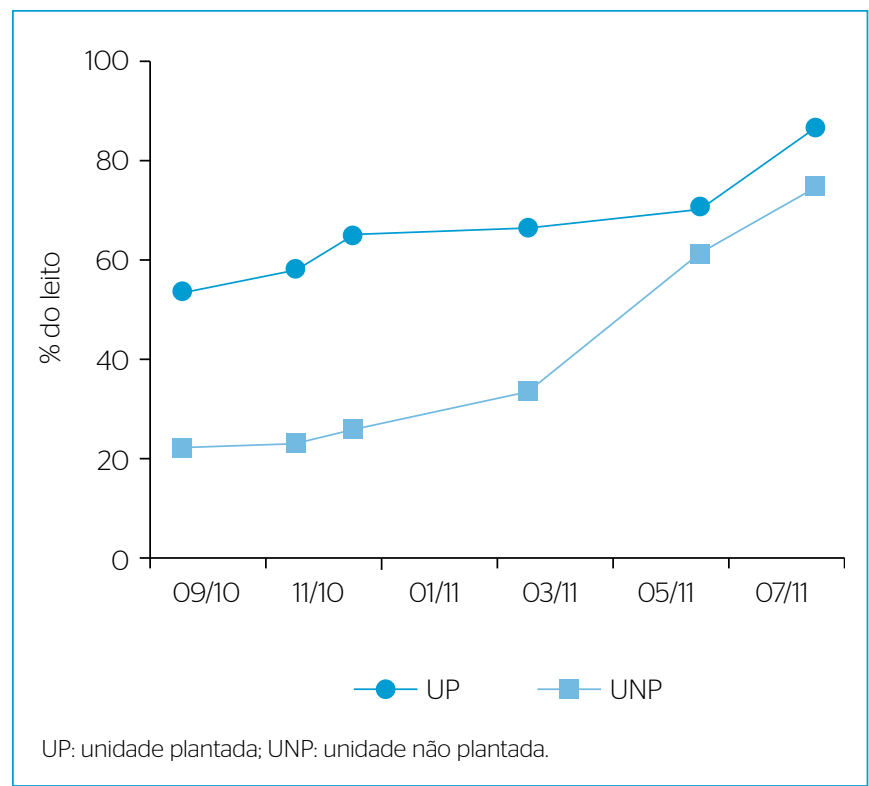

Figura 7 - Porcentagem do comprimento das unidades plantada e não plantada que apresentou escoamento superficial após quatro anos. 


\section{CONCLUSÃO}

Os resultados levam a concluir que as UP e UNP do SAC de escoamento horizontal subsuperficial tratando efluente de reator UASB apresentaram ótimo desempenho na remoção da matéria orgânica e sólidos em suspensão, durante os quatro anos de operação, com elevado grau de atendimento aos padrões de lançamento do estado de Minas Gerais (100\% de atendimento para DQO e SST nas UP e UNP). No entanto, a remoção do $\mathrm{N}$ total Kjeldahl e $\mathrm{N}$ amoniacal em ambas foi baixa.

Observou-se, como esperado, que a eficiência dos SACs calculada com base na carga removida foi maior do que a com base na concentração, em virtude da perda de água no sistema. As eficiências obtidas evidenciam resultados compatíveis com sistemas mais avançados de tratamento.

Durante o período de 4 anos de operação do sistema, a média da vazão efluente na UP foi de $6,6 \mathrm{~m}^{3} \cdot \mathrm{d}^{-1}$, e na UNP, de $6,8 \mathrm{~m}^{3} \cdot \mathrm{d}^{-1}$, tendo como perda média de água 19,5 e 15,0\% respectivamente. Vale ressaltar que essas perdas por evapotranspiração na UP e por evaporação na UNP influenciam o valor das concentrações efluentes.

Por meio do teste não paramétrico entre variáveis dependentes Wilcoxon a 5\% de significância, constatou-se que não houve diferença significativa nas concentrações efluentes entre as UP e UNP para a maior parte dos parâmetros estudados (DBO, DQO, SST e $\mathrm{N}$ total), exceto o $\mathrm{N}$ amoniacal.

Medições do perfil longitudinal da concentração de DQO indicaram contínuo decaimento ao longo do comprimento, com comportamento similar para as UP e UNP. A utilização do modelo convencional de fluxo em pistão com cinética de primeira ordem conduziu a um bom ajuste dos dados experimentais, e o valor obtido para o coeficiente de remoção de DQO na UP $\left(K=0,81 \mathrm{~d}^{-1}\right)$ está dentro da faixa usual reportada na literatura.

Apesar dos vários pontos positivos encontrados no desempenho das unidades, deve-se registrar que, ao longo dos quatro anos, observou-se expressivo escoamento superficial em ambas.

De forma global, e tendo por base os resultados obtidos nesse monitoramento de longo prazo, conclui-se que SACs de escoamento horizontal subsuperficial constituem uma ótima alternativa para o pós-tratamento de efluentes anaeróbios.

\section{AGRADECIMENTOS}

Os autores agradecem aos seguintes órgãos e instituições: CNPq, Capes, Finep, Fapemig, Funasa e Copasa.

\section{REFERÊNCIAS}

ALMEIDA, R.A.; POSCH, S.; PEREIRA, L.S.; GODINHO, C.A.; OLIVEIRA, L.F.C.; KLIEMANN, H.J. (2005) Tratamento de esgoto com plantas: sistema zona de raízes. In: CONSELHOREGIONAL DE ENGENHARIA ARQUITETURA E AGRONOMIA DO ESTADO DE GOIÁS. Prêmio CREA-Goiás de Meio Ambiente, 2004: compêndio dos trabalhos premiados. Goiânia: Conselho Regional de Engenharia Arquitetura e Agronomia do Estado de Goiás. p. 115-134.

AMERICAN PUBLIC HEALTH ASSOCIATION (APHA); AMERICAN WATER WORKS ASSOCIATION (AWWA); WATER ENVIRONMENT FEDERATION (WEF). (2005) Standard Methods for the Examination of Water and Wastewater. 21. ed. Washington, D.C.: APHA; AWWA; WEF. 953 p.

BASKAR, G.; DEEPTHA, V.T.; ANNADURAI, R. (2014) Comparison of treatment performance between constructed wetlands with different plants. International Journal of Research in Engineering and Technology, v. 3, p. 210-214.

BASTOS, R.K.X.; CALIJURI, M.L.; BEVILACQUA, P.D.; RIOS, E.N.; DIAS, E.H.O.; CAPELETE, B.C.; MAGALHÃES, T.B. (2010) Post-treatment of UASB reactor effluent in waste stabilization ponds and in horizontal flow constructed wetlands: a comparative study in pilot scale in Southeast Brazil. Water Science \& Technology, v. 61, n. 4, p. 995-1002.

BLANC, E.; QUIRION, P.; STROBL, E. (2008) The climatic determinants of cotton yields: evidence from a plot in West Africa. Agricultural and Forest Meteorology, v. 148, p.1093-1100.

BOUTILIER, L:; JAMIESON, R.; GORDON, R.; LAKE, C.; HART, W. (2OO9) Adsorption, sedimentation, and inactivation of E. coli within wastewater treatment wetlands. Water Research, New York, v. 43, p. 4370-4380.
BRASIL, M.S. (2005) Desempenho de sistema alagado construido para tratamento de esgoto doméstico. Tese (Doutorado) Universidade Federal de Viçosa, Viçosa, 2005.

CONLEY, L.M.; DICK, R.I.; LIOW, L.W. (1991) An assessment of the root zone method of wastewater treatment. Research Journal of the WPCF, v. 63, n. 3, p. 239-247.

DAVIES, L.; VICENTE, P.; RODRIGUES, F.; NOVAIS, J.; MARTINSDIAS, S. (2004) Degradação de um corante azo num leito piloto vertical plantado com Phragmites sp. Lisboa, Portugal: Centro de Engenharia Biológica e Química, Instituto Superior Técnico.

DAVISON, L.; HEADLEY, T.; PRATT, K. (2004) Performance and sustainability of small horizontal flow wetlands. In: INTERNATIONAL CONFERENCE ON WASTE STABILISATION PONDS, 6., and INTERNATIONAL CONFERENCE ON WETLAND SYSTEMS FOR WATER POLLUTION CONTROL, 9., 2004. Proceedings... Avignon, France: IWA/Astee. CD-ROM

DE PAOLI, A.C. (2010) Análise de desempenho e comportamento de wetlands horizontais de fluxo subsuperficial baseado em modelos hidráulicos e cinéticos. Dissertação (Mestrado) - Programa de Pós-graduação em Saneamento, Meio Ambiente e Recursos Hídricos, Universidade Federal de Minas Gerais, Belo Horizonte.

DE PAOLI, A.C:; VON SPERLING, M. (2010) Comparison between simple performance models applied to planted and unplanted subsurface-flow constructed wetlands for the post-treatment of UASB reactor effluents. In: INTERNATIONAL CONFERENCE ON WETLAND SYSTEMS FOR WATER POLLUTION CONTROL, 12 Veneza, Itália: IWA. 
DE PAOLI, A.C.; VON SPERLING, M. (2011) Avaliação da colmatação em wetlands de escoamento horizontal subsuperficial: comportamento dos sólidos e da condutividade hidráulica. In: CONGRESSO BRASILEIRO DE ENGENHARIA SANITÁRIA E AMBIENTAL, 26., 2011, Porto Alegre. Anais eletrônicos...

DORNELAS, F.L. (2008) Avaliação do desempenho de wetlands horizontais subsuperficiais como pós-tratamento de efluentes de reatores UASB. Dissertação (Mestrado) - Programa de Pós-graduação em Saneamento, Meio Ambiente e Recursos Hídricos, Universidade Federal de Minas Gerais, Belo Horizonte.

FIA, F.R.L. (2009) Modelos de remoção de matéria orgânica e nutrientes de águas residuárias da suinocultura em sistemas alagados construidos. Tese (Doutorado) - Universidade Federal de Viçosa, Viçosa.

KADLEC, R.H.; WALLACE, S.D. (2009) Treatment Wetlands. 2. ed. Boca Raton, Florida: CRC Press.

KASEVA, M.E. (2004) Performance of a sub-surface flow constructed wetland in polishing pre-treated wastewater - a tropical case study. Water Research, v. 38, p. 681-687.

MANDER, U.; LÕHMUS, K.; KUUSEMETS, V.; TEITER, S.; NURK, K. (2004) Dynamics of nitrogen and phosphorus budgets in a horizontal subsurface flow constructed wetland. In: INTERNATIONAL CONFERENCE ON WASTE STABILISATION PONDS, 6., INTERNATIONAL CONFERENCE ON WETLAND SYSTEMS FOR WATER POLLUTION CONTROL, 9. Avignon, France: IWA/Astee. CD-ROM.

MBULIGWE, S.E. (2004) Comparative effectiveness of engineered wetland systems in the treatment of anaerobically pre-treated domestic wastewatwer. Ecological Engineering, v. 23, p. 269-284.

METCALF; EDDY. (1991) Wastewater Engineering: treatment, disposal, reuse. 3. ed. New York: McGraw-Hill International.

MINAS GERAIS. (2008) Deliberação Normativa Conjunta COPAM/CERH-MG no 1, de 5 de Maio de 2008. Dispõe sobre a classificação dos corpos de água e diretrizes ambientais para o seu enquadramento, bem como estabelece as condições e padrões de lançamento de efluentes, e dá outras providências. Belo Horizonte: Conselho de Política Ambiental de Minas Gerais (COPAM); Conselho Estadual de Recursos Hídricos $(\mathrm{CERH})$.

PRESZNHUK, R.A.O; VAN KAICK, T.S.; CASAGRANDE JR., E.F; UMEZAWA, H.A. (2003) Tecnologia apropriada e saneamento: análise de eficiência de estações de tratamento de esgoto por meio de zona de raízes. In: SEMANA DE TECNOLOGIA: TECNOLOGIA PARA QUEM E PARA QUÊ? UM OLHAR INTERDISCIPLINAR. Atas... Curitiba: Editora Cefet-PR. 5 p. Disponível em: <www.ppgte.cefetpr. br/semanatecnologia/comunicacoes/tecnologia_ apropriada_e. pdf>. Acesso em: 01 nov. 2010.

REED, S.C.; CRITES, R.; MIDDLEBROOKS, E.J. (1988) Natural Systems for Waste Management and Treatment. New York: McGraw-Hill.

RIBEIRO, J.P.L. (2007) Modelação do comportamento hidráulico de leitos de macrófitas - estudo referenciado em dados de instalações protótipo em Portugal. Dissertação (Mestrado) - Instituto Superior Técnico, Universidade Técnica de Lisboa, Portugal. 104 p.
RIOS, D.A.; VÉLEZ, A.F.T.; PEÑA, M.R.; PARRA, C.A.M. (2009) Changes of flow patterns in a horizontal subsurface flow constructed wetland treating domestic wastewater in tropical regions. Ecological Engineering, v. 35, p. 274-280.

ROUSSEAU, D.P.L.; VANROLLEGHEM, P.A.; PAUW, N.D. (2004) Model-based design of horizontal subsurface flow constructed treatment wetlands: a review. Water Research, v. 38, p. 1484-1493.

SANDOVAL-COBO, J.J.; PEÑA, M.R. (2007) Análisis del desempeño de un humedal artificial de flujo sub-superficial en zonas tropicales basado en modelos hidráulicos y una cinética de primer orden. In: SEMINARIO MANEJO INTEGRAL DE AGUAS RESIDUALES DOMÉSTICAS - CONFERENCIA LATINO AMERICANA. Cali, Colombia.

SCHULZ, G. (2009) Sistema de Tratamento de Efluentes com Plantas Aquáticas Emergentes (PAE) para o Processo de Parboilização de Arroz. Dissertação (Mestrado) - Programa de Pós-Graduação em Engenharia, Canoas. 72 p.

SOLANO, M.L.; SORIANO, P.; CIRIA, M.P. (2004) Constructed wetlands as a sustainable solution for wastwater treatment in small villages. Biosystems Engineering, v. 87, n. 1, p. 109-118.

SOUSA, J.T.; HAANDEL, A.C.; LIMA, E.P.; GUIMARÃES, A.V. (2002) Desempenho de wetland construído tratando esgotos digeridos anaerobicamente. In: SIMPÓSIO LUSO BRASILEIRO DE ENGENHARIA SANITÁRIA E AMBIENTAL, 10., 16 a 19 de set. de 2002, Braga, Portugal. Anais... CD-ROM.

SOUSA, J.T.; VAN HAANDEL, A.; LIMA, E.P.C.; HENRIQUE, I.N. (2004) Utilização de wetland construído no pós-tratamento de esgotos domésticos pré-tratados em reator UASB. Engenharia Sanitária e Ambiental, v. 9, n. 4, p. 285-290.

STEIN, O.R.; BIEDERMAN, J.A.; HOOK, P.B.; ALLEN, W.C. (2006) Plant species and temperature effects on the $\mathrm{K}^{-C^{*}}$ first-order model for COD removal in batch-loaded SSF wetlands. Ecological Engineering, v. 26, p. 100-112

TONIATO, J.V.; ROQUE, O.C.C.; KACZOLA, F.; NASCIMENTO, V.B.; ROQUE, D.C. (2005) Avaliação de um wetland construído no tratamento de efluentes sépticos. In: CONGRESSO BRASILEIRO DE ENGENHARIA SANITÁRIA E AMBIENTAL, 23. Anais... Campo Grande. p. 11.

U. S. ENVIRONMENTAL PROTECTION AGENCY (USEPA). (1999) Constructed wetlands treatments of municipal wastewaters. EPA/625/r-99/010. Cincinnati, Ohio: USEPA. 152 p.

VALENTIM, M.A.A. (2003) Desempenho de leitos cultivados ("construted wetland") para tratamento de esgoto: contribuições para concepção e operação. Tese (Doutorado) - Faculdade de Engenharia Agrícola, Universidade Estadual de Campinas, Campinas. 210 p.

VICZNEVSKI, I.S.; MARCHESINI, G.B. (2002) Programa de saneamento rural: tratamento biológico de esgoto doméstico por zona de raízes. Joinville: Secretaria da Agricultura e Meio Ambiente/ Fundação Municipal 25 de Julho. Fôlder. 1 p.

VYMAZAL, J. (2004) Removal of phosphorus via harvesting of emergent vegetation in constructed wetlands for wastewater treatment. In: INTERNATIONAL CONFERENCE ON WASTE STABILISATION PONDS, 6., and INTERNATIONAL CONFERENCE ON WETLAND SYSTEMS FOR WATER POLLUTION CONTROL, 9. Avignon, France: IWA/Astee. Proceedings... CD-ROM. 